\title{
The impact of COVID-19 pandemic on health-related quality of life of adults visiting emergency departments and primary care settings in Alberta
}

\author{
Fatima Al Sayah ${ }^{1} \cdot$ Markus Lahtinen $^{2} \cdot$ Roland Simon $^{2} \cdot$ Benjamin Higgins ${ }^{2} \cdot$ Arto Ohinmaa $^{1} \cdot$ Jeffrey A. Johnson ${ }^{1}$
}

Received: 4 March 2021 / Accepted: 16 December 2021 / Published online: 11 January 2022

(c) The Author(s) under exclusive license to The Canadian Public Health Association 2022

\begin{abstract}
Objectives To examine the impact of COVID-19 pandemic on health-related quality of life (HRQL) of adults visiting emergency departments (ED) and primary care (PC) settings in Alberta, Canada, and explore whether this impact varies across demographic subgroups.

Methods Data from two repeated cross-sectional surveys that measured HRQL using EQ-5D-5L were used; "pre-COVID" Sept 2019-Feb 2020 (ED, N=5927; PC, N=317), "Wave-1" Mar 2020-Aug 2020 (ED, N=4781; PC, N=375), and "Wave-2" Sept 2020-Jan 2021 (ED, $N=4443$; PC, $N=327$ ).

Results In the ED sample, there were decrements in mild-extreme problems of $3.7 \%$ in mobility and $4.1 \%$ in usual activities from pre-COVID to wave 2 . There were very minor changes in mild-extreme problems in self-care (decrement $=1.3 \%$ ), pain/discomfort (decrement $=2.6 \%$ ), and anxiety/depression (decrement $=0.9 \%$ ). In the PC sample, there were increases of $4.8 \%$ in mild-extreme pain/discomfort and $10.7 \%$ in anxiety/depression from pre-COVID to wave 2. Despite these changes, HRQL of both samples pre-COVID and during waves 1 and 2 was worse than that of the general Alberta population. There were no significant variations in the impact of COVID-19 pandemic on HRQL across age, sex, and income subgroups in the ED survey; however, such variations were observed in the PC survey whereby younger adults, females, and those with high income had the largest HRQL deteriorations.

Conclusion The impact of COVID-19 pandemic on HRQL was minimal in adults seeking ED care, but more pronounced in those seen in PC, especially in terms of mental health. Policies around COVID-19 should take into account the needs of certain groups of the population, especially women and young people.
\end{abstract}

\section{Résumé}

Objectifs Examiner l'impact de la pandémie de COVID-19 sur la qualité de vie liée à la santé (QVLS) des adultes visitant les services d'urgence (SU) et les établissements de soins primaires (SP) en Alberta, au Canada, et déterminer si cet impact varie selon les sous-groupes démographiques.

Méthodes Les données de deux enquêtes transversales répétées qui ont mesuré la QVL à l'aide de l'EQ-5D-5L ont été utilisées; «pré-COVID » septembre 2019-février 2020 (SU, N=5 927; SP, N=317), « Vague-1 » mars 2020-août 2020 (SU, $N=4$ 781; SP, $N=375$ ) et « Vague-2 » septembre 2020-janvier 2021 (SU, $N=4$ 443; SP, $N=327$ ).

Résultats Dans l'échantillon du SU, il y a eu des diminutions des problèmes légers à extrêmes de 3,7 \% dans la mobilité et de $4,1 \%$ dans les activités habituelles de la période pré-COVID à la vague 2 . Il y a eu des changements très mineurs dans les problèmes légers à extrêmes dans les soins personnels (diminution $=1,3 \%$ ), douleur/gêne (diminution=2,6\%) et anxiété/ dépression (diminution=0,9\%). Dans l'échantillon SP, il y a eu des augmentations de 4,8\% de la douleur/gêne légère à extrême et de 10,7 \% de l'anxiété/de la dépression de la période pré-COVID à la vague 2. Malgré ces changements, la QVLS des deux échantillons avant la COVID et pendant les vagues 1 et 2 était pire que celle de la population générale de l'Alberta. Il n'y avait pas de variations significatives de l'impact de la pandémie de COVID-19 sur la QVLS selon l'âge, le sexe et les sous-groupes de revenu dans l'enquête SU; cependant, de telles variations ont été observées dans l'enquête SP, où les jeunes adultes, les femmes et les personnes à revenu élevé présentaient les plus fortes détériorations de la QVLS.

Extended author information available on the last page of the article 
Conclusion L'impact de la pandémie de COVID-19 sur la QVLS était minime chez les adultes cherchant des SU, mais plus prononcé chez ceux observés dans le SP, en particulier en termes de santé mentale. Les politiques autour de COVID-19 devraient prendre en compte les besoins de certains groupes de la population, en particulier les femmes et les jeunes.

Keywords COVID-19 pandemic $\cdot$ Health-related quality of life $\cdot$ EQ-5D-5L $\cdot$ Population-based surveys

Mots-clés Pandémie de COVID-19 · qualité de vie liée à la santé · EQ-5D-5L · enquêtes populationnelles

\section{Introduction}

The novel coronavirus disease (COVID-19) pandemic is recognized as a global health concern that has set public health institutions on high alert. Throughout the pandemic, countries have employed various measures to control the spread of the virus and minimize its impact (Anderson et al., 2020). In addition to promoting precautionary measures, public health interventions focused primarily on creating physical separation between people to reduce and delay transmission of the virus (Wang et al., 2020a). In Canada, COVID19 measures included closures of schools, daycares and all academic institutions, prohibitions on gatherings, closures of nonessential businesses, travel restrictions, and mandatory self-isolation for travelers (Detsky \& Bogoch, 2020).

In addition to the fear of infection, strategies of physical distancing may inadvertently increase social isolation and loneliness and limit social support for many individuals (Brooks et al., 2020). This in turn could have significant short- and long-term effects on physical, mental, and emotional health of the overall population (Brooks et al., 2020; Holmes et al., 2020). Two recent systematic reviews and meta-analyses on the psychosocial impact of COVID-19 in the general population reported a prevalence of $29.6 \%$ for stress, $31.9 \%$ for anxiety, and $33.7 \%$ for depression in one (Salari et al., 2020), and a pooled prevalence of anxiety and depression of $33 \%$ and $28 \%$, respectively in the other (Luo et al., 2020). Another systematic review reported relatively high rates of anxiety (6.33-50.9\%), depression (14.6-48.3\%), post-traumatic stress disorder (7-53.8\%), psychological distress (34.43-38\%), and stress (8.1-81.9\%) in the general population (Xiong et al., 2020). Female sex, younger age group ( $\leq 40$ years), presence of chronic/psychiatric illnesses, unemployment, and frequent exposure to social media/news concerning COVID-19 were associated with higher rates of distress (Xiong et al., 2020).

Emerging evidence suggests that mental health and well-being in Canada worsened during the pandemic. The Canadian Mental Health Index report released in July 2020 reported an overall 10-point drop in the Mental Health Index from the pre-pandemic benchmark in Canada, with the largest drop of -13.4 points observed in Alberta (Shepell, 2020). In another study, intense public health measures were found to be associated with increased psychosocial distress, including elevated levels of overall distress, panic, emotional disturbances, and depression (Serafini et al., 2020). Statistics Canada examined the impact of COVID-19 pandemic on over 200,000 Canadians, and found that during the pandemic 6 in 10 seniors reported to be very or extremely concerned about their own health, young Canadians are concerned about social and economic effects of the pandemic, and women are concerned about violence at home (Statistics Canada, 2020).

Given the immense burden that the pandemic is placing on individuals' physical, mental, and social functioning, it is imperative to explore its impact on health-related quality of life (HRQL) of populations to inform COVID-19-related policies and strategies, including social distancing, lockdowns, quarantine, and others. This study aimed to examine the impact of COVID-19 pandemic on HRQL of adults in the province of Alberta using survey data from emergency departments (ED) and primary care (PC) settings, and explore whether this impact varies across demographic groups of the population.

\section{Methods}

\section{Context}

The first case of COVID-19 in Alberta was detected on March 5, 2020, with a total of 327,283 cases as of November 8 , 2021 (Government of Alberta, 2021). The first wave of the pandemic continued until the end of September 2020 with a total of 18,206 cases. The number of cases started increasing in October marking the start of the second wave, which was much more detrimental than the first with a total of 115,584 cases recorded during wave 2 . The initial pandemic response was instated in March 2020 with the most stringent lockdown measures, including closure of schools, universities and nonessential businesses, travel restrictions, restrictions of social gatherings, and invoking the Quarantine Act. Stage 1 of re-opening started in mid-May and continued until mid-June, followed by stage 2 which lasted until November. With the surge of cases in November, some restrictions were re-instated, followed by a full lockdown in December, which remained in place until the beginning of February 2021. Throughout the pandemic, the Governments of Alberta and Canada have implemented substantial economic relief measures, including 
direct monetary benefits, home mortgage deferrals, benefits for individuals with special needs, students, and older adults, as well as support for small and moderate businesses.

\section{Data source}

Data from two repeated cross-sectional surveys conducted by Health Quality Council of Alberta (HQCA) were used in this study:

- Emergency Department Patient Experience of Care Survey: Data collection was done via a computer-assisted telephone interviewing system via phone at a median of 3 weeks after an ED visit. Patients were excluded if they left the ED without being seen or treated, died in the context of their ED or inpatient stay, had no contact information, or had privacy-sensitive cases.

- Primary Care Patient Experience Survey: Respondents to the ED survey who consented to participating in future research were contacted to participate in this survey. Data collection was done via an online survey. Respondents who have a regular family doctor, and have visited that doctor at least once in the past 12 months were eligible to participate.

In both surveys, a HRQL measure - the EQ-5D-5L—was administered to all participants. This data source was selected for this analysis as both surveys continued to be conducted throughout the COVID-19 pandemic and as such data on HRQL from different waves of the pandemic can be obtained. COVID-19 periods were defined as: "pre-COVID" September 2019 - February 2020; "wave 1" March 2020 - August 2020; and "wave 2" September 2020 - January 2021. Ethics approval for this study was obtained from the University of Alberta Health Research Ethics Board (ID Pro00101660).

\section{Measures}

The EQ-5D-5L is one of the most commonly used generic preference-based measures of HRQL. It includes a descriptive system that captures five dimensions of health: mobility, selfcare, usual activities, pain/discomfort, and anxiety/depression; each with five levels of problems ranging from none (level 1) to extreme (level 5), describing 3125 distinct health states (Herdman et al., 2011). Due to small sample sizes in levels 4 and 5, we grouped the levels into two (level 1= "no problems"; levels $2-5=$ "present problems") and three modalities (level $1=$ "no problems"; levels $2-3=$ "mild-moderate problems"; levels 4-5= "severe-extreme problems") for each dimension; results from both were similar, and as such we report those from the former. An index score was calculated using health state preferences of the general Canadian population (Xie et al., 2016). The EQ-5D-5L also includes a visual analogue scale
(VAS) that assesses overall health on a scale ranging from 0 "worst imaginable health" to 100 "best imaginable health". Demographic data were collected in both surveys and included age, sex, highest level of education, and total annual household income. Additionally, respondents completed a self-rated health question on a 5-point Likert scale ranging from excellent to poor.

\section{Data analysis}

First, we compared demographic characteristics of participants in each COVID-19 period. Then, we examined EQ5D-5L dimensions, index, and VAS scores pre-COVID and during waves 1 and 2 in the overall sample compared to Alberta EQ-5D-5L population norms, and by subgroups defined by age, sex, and total annual household income. Samples' characteristics in the three COVID-19 periods were compared with those of the Alberta population norms sample (Alberta PROMs and EQ-5D, 2018). Bivariate analyses using chi-square test, $t$-test, or one-way ANOVA were used to test differences in HRQL between COVID-19 periods as appropriate. Minimal important difference (MID) of 0.04 for EQ-5D-5L index score (McClure et al., 2017) and 7.0 for the VAS score (Pickard et al., 2007) were used to determine an important difference. Data analysis was conducted using STATA 15.0; $p$ values less than 0.05 were considered statistically significant.

\section{Results}

There was a fair distribution of ED and PC survey respondents across age groups, sex, education, and income levels pre-COVID and during waves 1 and 2 (Table 1). There were no differences in participant characteristics across COVID19 periods in the PC survey, and slight but statistically significant differences in education and self-reported health in the ED survey.

The ED samples had fewer respondents aged 45-64 years and more aged $65+$ years, and more males compared to the Alberta population norms sample (supplementary Table 1). The PC samples had fewer respondents younger than 44 years and more 45 years and older, and more females compared to the norms sample. Overall, EQ-5D-5L index and VAS scores were lower in the ED and PC survey samples compared to the norms sample. The ED samples had higher rates of reporting problems in mobility, self-care, and usual activities, but lower rates for pain/discomfort and similar rates for anxiety/depression compared to the norms sample, while the PC samples had higher rates of reporting problems in all dimensions.

In the ED survey, there was a $3.7 \%$ decrease in the proportion of respondents reporting problems in mobility 
Table 1 Demographic characteristics of respondents in ED and PC surveys across COVID-19 periods

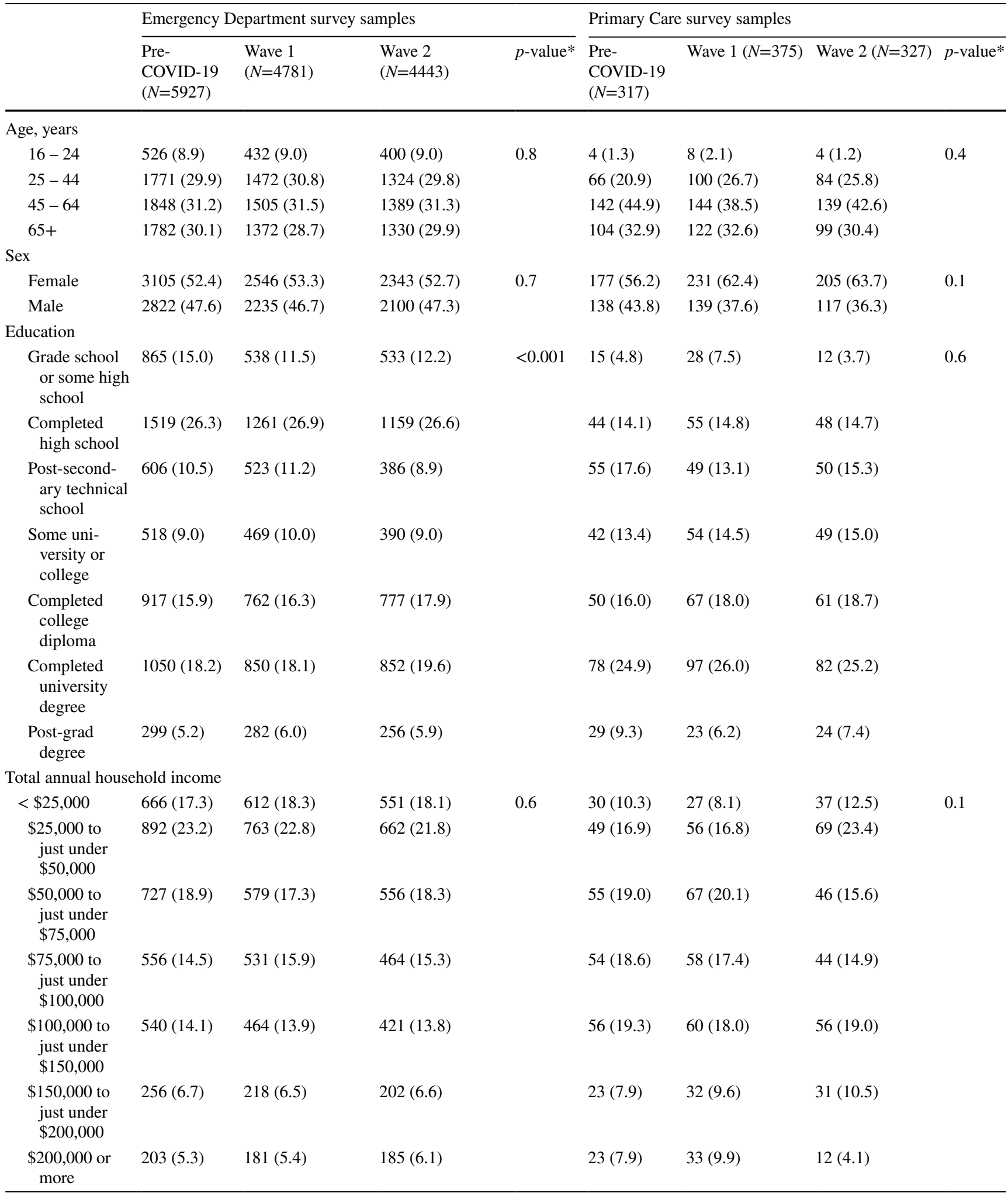

$(p<0.001), 1.3 \%$ in self-care $(p=0.1), 4.1 \%$ in usual activities $(p<0.001), 2.6 \%$ in pain/discomfort $(p=0.01)$, and a $0.9 \%$ increase in anxiety/depression $(p=0.3), 0.01$ in the index $(p=0.01)$, and 2.1 in the VAS $(p=0.1)$ scores from pre-COVID to wave 2 (Figure 1). In the PC survey, there was a $1 \%$ decrease in the proportion of respondents reporting 
Table 1 (continued)

\begin{tabular}{|c|c|c|c|c|c|c|c|}
\hline \multicolumn{4}{|c|}{ Emergency Department survey samples } & \multicolumn{4}{|c|}{ Primary Care survey samples } \\
\hline $\begin{array}{l}\text { Pre- } \\
\text { COVID-19 } \\
(N=5927)\end{array}$ & $\begin{array}{l}\text { Wave } 1 \\
(N=4781)\end{array}$ & $\begin{array}{l}\text { Wave } 2 \\
(N=4443)\end{array}$ & $p$-value* & $\begin{array}{l}\text { Pre- } \\
\text { COVID-19 } \\
(N=317)\end{array}$ & Wave $1(N=375)$ & Wave $2(N=327)$ & $e^{*}$ \\
\hline
\end{tabular}

Overall self-reported health

$\begin{array}{llllllll}\text { Poor } & 549(9.3) & 439(9.3) & 351(8.0) & 0.01 & 103(32.6) & 100(26.8) & 94(28.3) \\ \text { Fair } & 1088(18.5) & 845(17.8) & 839(19.0) & & 87(27.5) & 123(33.0) & 118(36.2) \\ \text { Good } & 2014(34.2) & 1596(33.7) & 1433(32.5) & & 77(24.4) & 98(26.3) & 79(24.2) \\ \text { Very good } & 1555(26.4) & 1301(27.5) & 1181(26.8) & & 26(8.2) & 25(6.7) & 18(5.5) \\ \text { Excellent } & 682(11.6) & 556(11.7) & 602(13.7) & & 23(7.3) & 27(7.2) & 17(5.2)\end{array}$

* $p$-value of chi-square test comparing demographic characteristics distribution across the three COVID-19 periods

problems in mobility $(p=0.9), 2.8 \%$ increase in self-care $(p=0.2), 3.6 \%$ decrease in usual activities $(p=0.7), 4.8 \%$ in pain/discomfort $(p=0.4), 10.7 \%$ increase in anxiety/depression $(p=0.02)$, and a 0.03 decrease in the index $(p=0.6)$, and 0.3 in the VAS $(p=0.6)$ scores from pre-COVID to wave 2 (Figure 2).

In both surveys and across all pandemic periods, EQ5D-5L dimensions, index, and VAS scores varied by demographic subgroups (supplementary Figures 1 and 2). In the ED survey (supplementary figure 1), significant changes in reporting mild-extreme problems in mobility from preCOVID to wave 2 were in those aged 16-24 years (decrement $=6.4 \% ; p=0.04$ ) followed by those aged $25-44$ years (decrement $=4.5 \% ; p=0.01$ ). For usual activities, statistically significant changes were in respondents aged 25-44 years $($ decrement $=5.3 \% ; p<0.001)$ and $45-64$ years $($ decrement $=5.1 \% ; p=0.02$ ). For pain/discomfort, significant changes were in those aged 16-24 years (decrement $=7.9 \%$; $p=0.04)$ and $25-44$ years (decrement $=4.5 \% ; p=0.01$ ), in females (decrement $=2.5 \% ; p=0.02$ ), and those with low $($ decrement $=3.8 \% ; p=0.04)$ or high income $($ decrement $=$ $3.9 \% ; p=0.03)$. Changes in the index score in all examined subgroups were statistically significant; however, the magnitude of these changes was smaller than the MID. There were no significant changes in self-care, anxiety/depression, or the VAS in all examined subgroups.

In the PC survey (supplementary Figure 2), there were no statistically significant changes in mobility, self-care, usual activities, pain/discomfort, or the VAS in all examined subgroups, although some changes are not negligible, e.g., for mobility, a decrement of $7.8 \%$ in respondents aged 16-44 years, $8.1 \%$ in males, $8.9 \%$ in those with the lowest income, and an $8.7 \%$ increase in those aged $45-64$ years. Similarly, for usual activities, there were decrements of $7.1 \%$ and $9.9 \%$ in those aged 16-44 years and in males, respectively; and for pain, there were increases of $7.4 \%, 6.8 \%, 9.2 \%$, and $7.0 \%$ in respondents aged 45-64 years, those aged $65+$ years, females, and those with the highest income, respectively.
The largest changes and differences between subgroups were observed in the anxiety/depression dimension whereby participants aged 16-44 years and those aged $45-64$ years had a respective $20.7 \%$ and $11.9 \%$ increase in reporting mild-extreme anxiety/depression from pre-COVID to wave 2. Similarly, females and males had respective increases of $11.4 \%$ and $10.2 \%$ and respondents with low, middle, and high income had increases of $14.4 \%, 9.0 \%$, and $8.4 \%$, respectively, in reporting mild-extreme anxiety/depression. Changes in the index score in females and those with high income were statistically significant; however, the magnitude of these changes was smaller than the MID.

\section{Discussion}

This study suggests that the COVID-19 pandemic and related public health measures had little impact on HRQL, as measured by the EQ-5D-5L, in adults attending EDs in Alberta, but rather more pronounced impact in those seeking PC services, particularly with respect to mental health. There was a slight improvement in health-related quality of life in the ED sample, primarily driven by small improvements in mobility and usual activities. This may be related to the nature of the COVID-19 pandemic-related restrictions that limited movement and changed the nature of people's usual activities. In the PC sample, the proportion of respondents reporting mild-extreme anxiety/depression increased from $50.5 \%$ pre-COVID to $58.4 \%$ during wave 1 and $61.2 \%$ during wave 2 , compared to an average of $37.2 \%$ in the general Alberta population before the COVID-19 pandemic. There were significant variations in the impact of COVID19 pandemic on health-related quality of life across age, sex, and income subgroups in the PC survey, but not in the ED survey.

There were no changes in one dimension and opposite changes in another that may explain the lack of change 
Mobility

50

40

40

30

20

10

0

P-value $<0.001$

Pre-COVID19 Wave 1 Wave 2

Usual activities

70

60

50

40

$\%$

20

10

P-value $<0.001$

Pre-CovID19
46.3

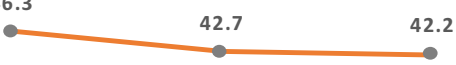

Alberta Norm 26\%

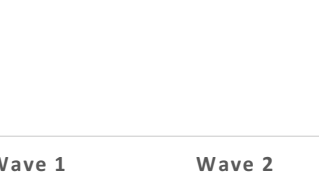

Self-care

30

$\% 20$

16.7 15.6 15.4

10

Alberta Norm 5.9\%

0 P-value $=0.1$

Pre-COVID19 Wave 1 Wave 2

Pain/discomfort

90

70

60

50

\% 40

30

20

10

P-value $=0.01$

Pre-COVID19 Wave 1 Wave 2

Anxiety/depression

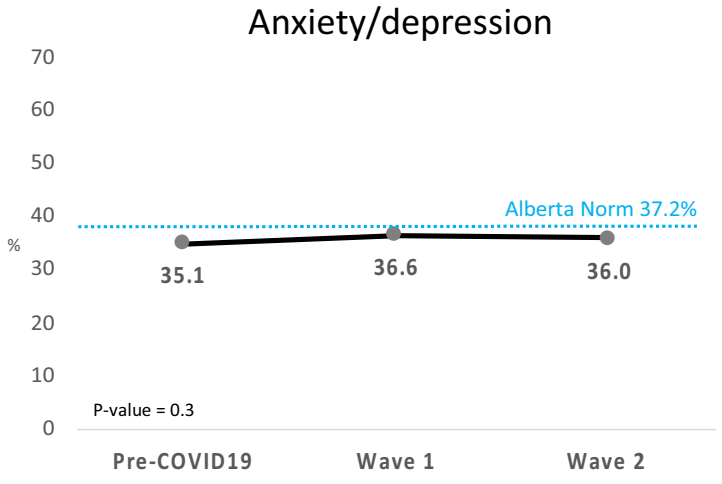

Index score

VAS score
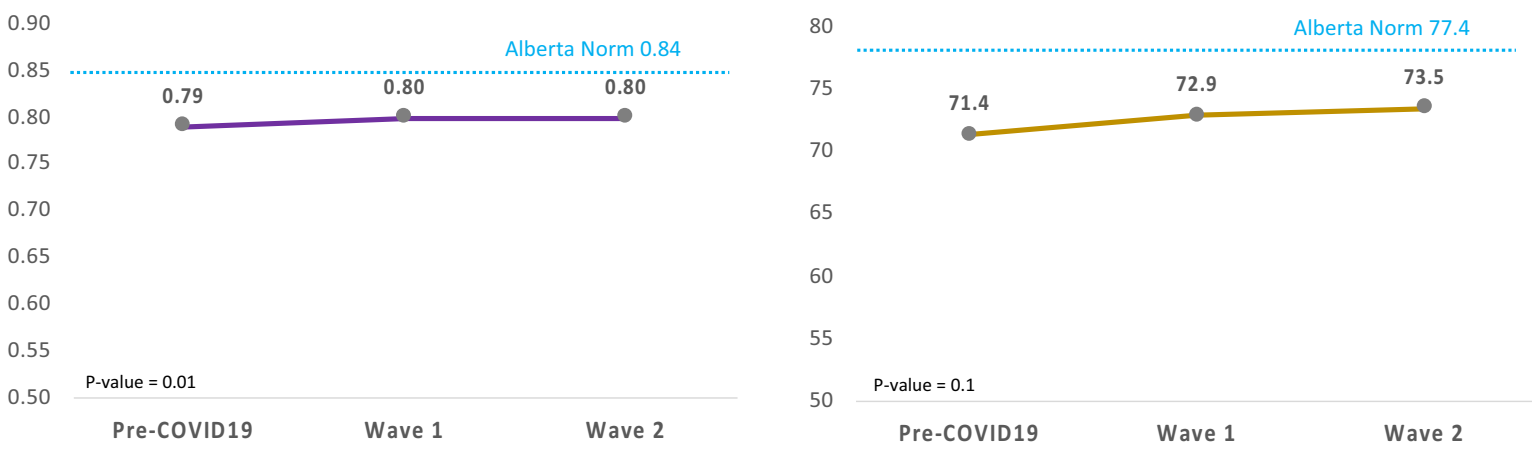
4Fig. 1 Proportion of mild-extreme problems (levels 2-5) in EQ5D-5L dimensions and index and VAS scores by COVID-19 period in the ED survey compared to Alberta population norms. VAS, visual analogue scale. Index score ranges from -0.149 to 0.949; VAS score ranges from 0 to 100 . $p$-values are for statistical tests comparing values across the three COVID-19 periods

in total scores in the ED survey sample. However, such changes, namely less problems in self-care and usual activities and more problems in pain/discomfort and anxiety/ depression, were observed in the PC survey, which may explain the lack of change in total scores. Additionally, the proportion of respondents reporting levels $2,3,4$, or 5 in all dimensions was consistent across the three time periods in both surveys (data not shown), suggesting no worsening of symptoms in those who already had problems.

Our findings are consistent with those in other countries where the impact of COVID-19 pandemic on psychosocial health and HRQL in the general population ranged from small to moderate, including China (Qiu et al., 2020; Wang et al., 2020b; Wang et al., 2020c; Zhang \& Ma, 2020), Hong Kong (Choi et al., 2020), United Kingdom (Chandola et al., 2020; Pierce et al., 2020; Shevlin et al., 2020), United States (Ettman et al., 2020; Kantor \& Kantor, 2020), Spain (González-Sanguino et al., 2020), Italy (Rossi et al., 2020), Germany (Röhr et al., 2020), Vietnam (Tran et al., 2020), and Japan (Suzuki et al., 2020). Additionally, the variation in the impact of the pandemic across population subgroups was also observed in other populations whereby females, younger adults, the unemployed, those with lower socioeconomic status, and those with higher risk for contracting COVID-19 were more likely to experience higher levels of psychosocial distress compared to other groups in the population (Luo et al., 2020; Xiong et al., 2020).

In Alberta, the first wave of the COVID-19 pandemic was well controlled, and Albertans received ample social and economic support from the provincial and federal governments. These supports and others, along with working from home for a large proportion of the population, may have acted as protective factors and led to alleviation of some problems, especially those in mobility and usual activities that appeared to have slightly improved in some respondent groups. Protective factors associated with a lower psychological impact of the outbreak include sufficient medical resources, up-to-date and accurate information, taking precautionary measures, trust in doctors, and low risk of contracting COVID-19 (Luo et al., 2020; Wang et al., 2020d). Although the second wave of COVID-19 in Alberta has been more severe and had an immense impact on socio-economic aspects of Albertans' life, as well as a huge burden on the healthcare system, it appears that these factors have not immensely impacted HRQL in adults seeking ED services; however, this impact was greatly present in the PC sample.
This may highlight unmeasured differences in the respondents between the two surveys.

The study results should be interpreted in light of some limitations. First, the study used two repeated cross-sectional surveys that include different participants in each administration, and as such, there were no repeated measures on the same individuals, prohibiting the examination of individual-level changes in HRQL due to the COVID-19 pandemic. Although these data may not be ideal for this analysis, no other sources of population-based data covering the pre-COVID period and the first waves of the pandemic in Alberta were available at the time of this study, and as such, this study offers an idea about the changes in HRQL in the population. Additionally, the same sampling methodology was followed in each administration of these surveys, which ensures relatively representative and fairly comparable samples across time. Second, the samples used in both surveys are not representative of the general population but rather of adults in the general population who seek ED or PC services; these individuals are likely to be different from those who do not seek these services. As such, the generalizability of the results is limited to similar groups of the population. Third, the surveys did not include measurements of factors that may be associated with HRQL or psychosocial impact of the pandemic. However, we could reflect on results from the HQCA COVID-19 survey $(N=11,149)$, which assessed the experience and impact of COVID-19 pandemic on adults in Alberta during wave 1 (Health Quality Council of Alberta, 2020). The survey found that Albertans have high trust in provincial health authorities as reliable sources of information. While $95 \%$ of respondents reported feeling reasonably to well protected from contracting COVID-19, $70 \%$ reported feeling more stressed since the beginning of the pandemic, $48 \%$ reported sleep problems, $46 \%$ reported feeling lonely, $48 \%$ were unable to exercise as normal, and $58 \%$ indicated they could not stay connected with family and friends due to restrictions. Additionally, $60 \%$ and $41 \%$ of respondents reported a worsening in their mental and physical health during the pandemic, respectively. These figures may explain the impact on mental health observed in the PC sample. The survey reported that $30 \%$ of Albertans delayed seeking healthcare during the pandemic; this may have impacted the characteristics of individuals presenting to ED or PC settings. Fourth, although the ED survey sample in the pre-COVID period was comparable to those in waves 1 and 2 in terms of demographics, there are other unmeasured characteristics (e.g., number of comorbidities) that may explain the lack of change we observed in this survey. This stands as one of the key limitations in this study. Similarly, respondents in both surveys could be different from the general Alberta population in unmeasured characteristics; this also limits the generalizability of these results to similar population groups. Finally, the usefulness of the EQ-5D-5L 


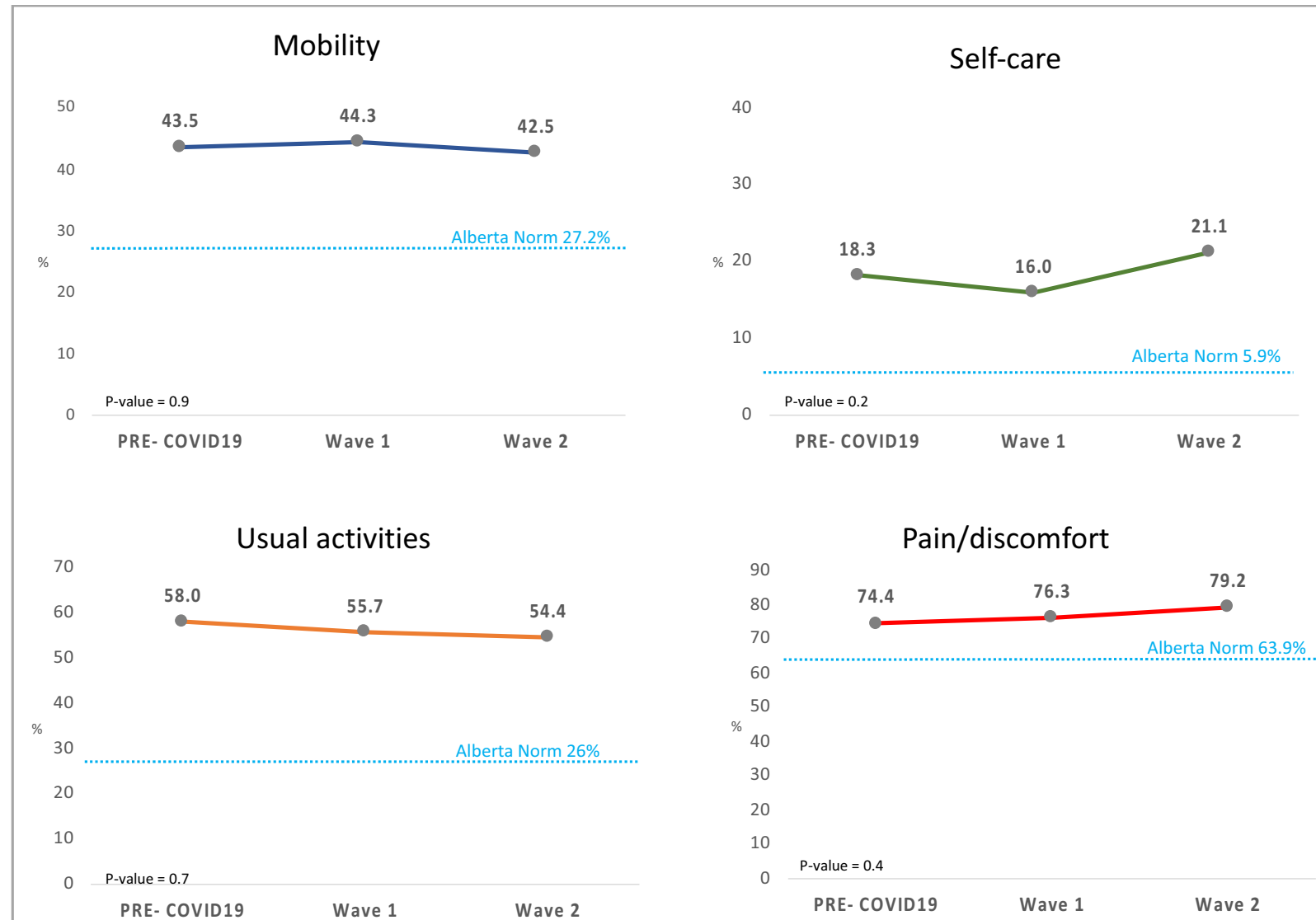

Anxiety/depression

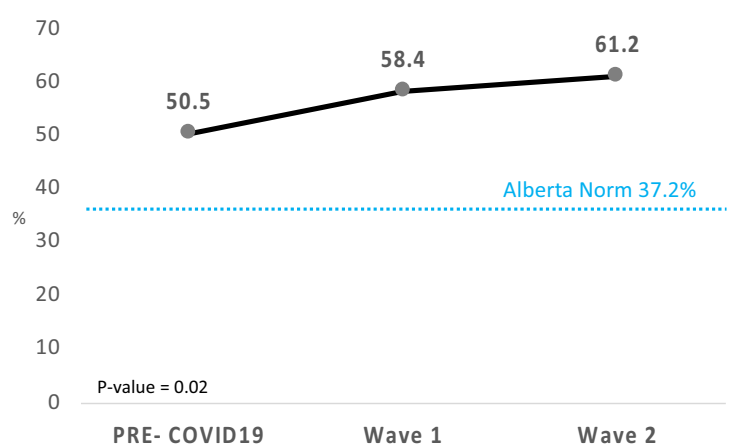

Index score

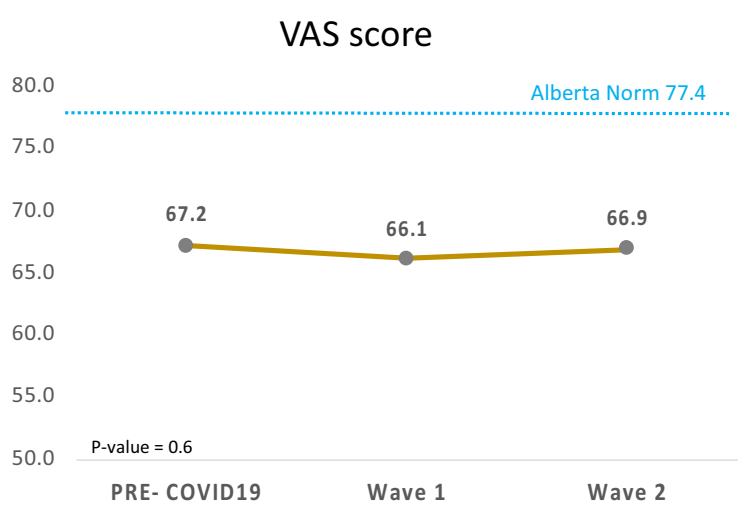


4Fig. 2 Proportion of mild-extreme problems (levels 2-5) in EQ5D-5L dimensions and index and VAS scores by COVID-19 period in primary care survey compared to Alberta population norms. VAS, visual analogue scale. Index score ranges from -0.149 to 0.949 ; VAS score ranges from 0 to 100 . $p$-values are for statistical tests comparing values across the three COVID-19 periods

instrument in capturing aspects of HRQL that are important to the general population in the context of COVID-19 pandemic should be considered. While the EQ-5D-5L captures five general aspects of health and includes an assessment of overall health, it does not focus on social functioning and other specific aspects of emotional health (e.g., distress, fear, worry about future) that may have been impacted during the pandemic. Furthermore, it seems that while some aspects of HRQL may improve (e.g., physical health), others (e.g., mental health) deteriorate; this highlights the importance of examining dimension and domain-level data in HRQL measures and not relying on total scores which may obscure such changes.

\section{Conclusion}

In adults seeking emergency and primary care services in Alberta, the impact of the COVID-19 pandemic on HRQL was minimal in the former and more pronounced in the latter, especially in terms of mental health, with noticeable variability across population subgroups. Policies around COVID-19 should take into account the needs of certain groups of the population, especially women and young people.

\section{Contributions to knowledge}

What does this study add to existing knowledge?

- This study highlights the impact of COVID-19 pandemic on physical and mental aspects of health-related quality of life (HRQL) in Alberta, by examining HRQL trends pre-COVID and throughout waves 1 and 2 in adults seeking emergency and primary care services. The impact was found to be minimal in the former and ranged from mild to moderate in the latter with significant variations across population subgroups, which suggests disparity in the impact of the pandemic in the population.

- Additionally, it seems that the pandemic led to improvements in certain aspects of HRQL (e.g., mobility) and deterioration in others (e.g., anxiety/depression).
What are the key implications for public health interventions, practice or policy?

- Policies, strategies, and measures aimed at controlling the pandemic should take into account the impact of the pandemic on various aspects of health, and the disproportionate impact in certain groups of the population, especially younger adults and women.

Supplementary Information The online version contains supplementary material available at https://doi.org/10.17269/ s41997-021-00606-4.

Author contributions All the authors contributed to the study conception and design. Data analysis was performed by Fatima Al Sayah with input from all co-authors on the analytical plan. The first draft of the manuscript was written by Fatima Al Sayah and all the authors commented on previous versions of the manuscript. All the authors read and approved the final manuscript.

Funding This study was funded by the EuroQol Research Foundation (grant number: 102-2020RA).

Data availability Data used in this study could be shared upon request upon approval from the Health Quality Council of Alberta (the custodian of the data).

Code availability Statistical codes used in the analysis could be shared upon request.

\section{Declarations}

Ethics approval The study procedures were approved by the Health Research Ethics Board at the University of Alberta.

Consent to participate All participants in both surveys used in this study provided informed consent.

Consent for publication Not applicable; there are no individual data or images in this manuscript.

Conflict of interest Fatima Al Sayah, Arto Ohinmaa, and Jeffrey A. Johnson are members of the EuroQol Group, the founder of the EQ-5D instrument. Markus Lahtinen, Roland Simon, and Benjamin Higgins have no conflicts of interest to declare.

\section{References}

Alberta PROMs and EQ-5D Research and Support Unit. (2018). Alberta Population Norms for EQ-5D-5L. Available from: https://apersu.ca/ wp-content/uploads/2021/02/Alberta-Norms-Report_APERSU.pdf

Anderson, R. M., et al. (2020). How will country-based mitigation measures influence the course of the COVID-19 epidemic? Lancet, 395(10228), 931-934. 
Brooks, S. K., et al. (2020). The psychological impact of quarantine and how to reduce it: Rapid review of the evidence. Lancet, 395(10227), 912-920.

Chandola, T., Kumari, M., Booker, C. L., Benzeval, M. (2020). The mental health impact of COVID-19 and lockdown-related stressors among adults in the UK. Psychol Med, 1-10.

Choi, E. P. H., Hui, B. P. H., \& Wan, E. Y. F. (2020). Depression and anxiety in Hong Kong during COVID-19. International Journal of Environmental Research and Public Health, 17(10).

Detsky, A. S., \& Bogoch, I. I. (2020). COVID-19 in Canada: Experience and response. JAMA, 324(8), 743-744.

Ettman, C. K., Abdalla, S. M., Cohen, G. H., Sampson, L., Vivier, P. M., Galea, S. (2020). Prevalence of Depression Symptoms in US Adults Before and During the COVID-19 Pandemic. JAMA Netw Open, 3(9)

González-Sanguino, C., et al. (2020). Mental health consequences during the initial stage of the 2020 coronavirus pandemic (COVID19 ) in Spain. Brain, Behavior, and Immunity, 87, 172-176.

Government of Alberta. COVID-19 Cases in Alberta [cited 2021; Available from: https://www.alberta.ca/covid-19-alberta-data. aspx. Accessed 8 Nov 2021

Health Quality Council of Alberta. (2020). COVID-19 experiences and impact survey: Voice of Albertans May - June 2020. Calgary, Alberta: Health Quality Council of Alberta.

Herdman, M., et al. (2011). Development and preliminary testing of the new five-level version of EQ-5D (EQ-5D-5L). Quality of Life Research, 20(10), 1727-1736.

Holmes, E. A., O’Connor, R. C., Perry, V. H., Tracey, I., Wessely, S., Arseneault, L., et al. (2020). Multidisciplinary research priorities for the COVID-19 pandemic: a call for action for mental health science. Lancet Psychiatry, 30168-1.

Kantor, B. N., Kantor, J. Mental health outcomes and associations during the COVID-19 pandemic: A cross-sectional population-based study in the United States. Front Psychiatry, 11

Luo, M., Guo, L., Yu, M., Jiang, W., Wang, H. (2020). The psychological and mental impact of coronavirus disease 2019 (COVID-19) on medical staff and general public - A systematic review and meta-analysis. Psychiatry Res, 291.

McClure, N., et al. (2017). Instrument-defined estimates of the minimally important difference for EQ-5D-5L index scores. Value in Health, 20(4), 644-650.

Pickard, A. S., Neary, M. P., \& SJ Cella, D. (2007). Estimation of minimally important differences in EQ-5D utility and VAS scores in cancer. Health and Quality of Life Outcomes, 5, 70.

Pierce, M., et al. (2020). Mental health before and during the COVID19 pandemic: A longitudinal probability sample survey of the UK population. Lancet Psychiatry, 7(10), 883-892.

Qiu, J., Shen, B., Zhao, M., Wang, Z., Xie, B., Xu, Y. (2020). A nationwide survey of psychological distress among Chinese people in the COVID-19 epidemic: implications and policy recommendations. Gen Psychiatr, 33(2).

Röhr, S., Reininghaus, U., Riedel-Heller, S. G. (2020). Mental wellbeing in the German old age population largely unaltered during COVID-19 lockdown: results of a representative survey. $B M C$ Geriatr, 20(1).

Rossi, R., Socci, V., Talevi, D., Mensi, S., Niolu, C., Pacitti, F., et al. (2020). COVID-19 pandemic and lockdown measures impact on mental health among the general population in Italy. Front Psychiatry, 11
Salari, N., Hosseinian-Far, A., Jalali, R., Vaisi-Raygani, A., Rasoulpoor, S., Mohammadi, M., et al. (2020). Prevalence of stress, anxiety, depression among the general population during the COVID-19 pandemic: a systematic review and meta-analysis. Global Health, 16(1).

Serafini, G., Parmigiani, B., Amerio, A., Aguglia, A., Sher, L., Amore, M. (2020). The psychological impact of COVID-19 on the mental health in the general population. QJM : monthly Journal of the Association of Physicians, 113(8):531-7.

Shepell, M. (2020). Canadians are feeling unprecedented levels of anxiety, according to Mental Health Index.

Shevlin, M., McBride, O., Murphy, J., Miller, J. G., Hartman, T. K., Levita, L., et al. (2020). Anxiety, depression, traumatic stress and COVID-19-related anxiety in the UK general population during the COVID-19 pandemic. BJPsych Open, 6(6).

Statistics Canada. (2020). Impacts of COVID-19 on Canadians: First results from crowdsourcing. Statistics Canada.

Suzuki, Y., Maeda, N., Hirado, D., Shirakawa, T., Urabe, Y. (2020). Physical activity changes and its risk factors among communitydwelling Japanese older adults during the COVID-19 Epidemic: Associations with subjective well-being and health-related quality of life. International Journal of Environmental Research and Public Health, 17(18).

Tran, B. X., Nguyen, H. T., Le, H. T., Latkin, C. A., Pham, H. Q., Vu, L. G., et al. (2020). Impact of COVID-19 on economic well-being and quality of life of the Vietnamese during the national social distancing. Front Psychol, 11.

Wang, C., Liu, L., Hao, X., Guo, H., Wang, Q., Huang, J., et al. (2020a). Evolving epidemiology and impact of non-pharmaceutical interventions on the outbreak of coronavirus disease 2019 in Wuhan, China. medRxiv.

Wang, C., Pan, R., Wan, X., Tan, Y., Xu, L., Ho, C. S., et al. (2020b). Immediate Psychological Responses and Associated Factors during the Initial Stage of the 2019 Coronavirus Disease (COVID-19) Epidemic among the General Population in China. Int J Environ Res Public Health, 17(5).

Wang, H., Xia, Q., Xiong, Z., Li, Z., Xiang, W., Yuan, Y., et al. (2020c). The psychological distress and coping styles in the early stages of the 2019 coronavirus disease (COVID-19) epidemic in the general mainland Chinese population: A web-based survey. PLoS One, 15(5).

Wang, C., Pan, R., Wan, X., Tan, Y., Xu, L., McIntyre, R. S., et al. (2020d). Longitudinal study on the mental health of general population during the COVID-19 epidemic in China. Brain Behav Immun, 87:40-8.

Xie, F., et al. (2016). A time trade-off-derived value set of the EQ5D-5L for Canada. Medical Care, 54(1), 98-105.

Xiong, J., et al. (2020). Impact of COVID-19 pandemic on mental health in the general population: A systematic review. Journal of Affective Disorders, 277, 55-64.

Zhang, Y., Ma, Z. F. (2020). Impact of the COVID-19 Pandemic on Mental Health and Quality of Life among Local Residents in Liaoning Province, China: A Cross-Sectional Study. Int J Environ Res Public Health, 17(7)

Publisher's note Springer Nature remains neutral with regard to jurisdictional claims in published maps and institutional affiliations. 


\section{Authors and Affiliations}

\section{Fatima Al Sayah ${ }^{1} \cdot$ Markus Lahtinen $^{2} \cdot$ Roland Simon $^{2} \cdot$ Benjamin Higgins $^{2} \cdot$ Arto Ohinmaa $^{1} \cdot$ Jeffrey A. Johnson ${ }^{1}$}

$\bowtie$ Fatima Al Sayah

falsayah@ualberta.ca

2 Health Quality Council of Alberta, Calgary, AB, Canada

1 Alberta PROMs and EQ-5D Research and Support Unit (APERSU), School of Public Health, University of Alberta, 2-040 Li Ka Shing Centre for Health Research Innovation, 8602 - 112 St, Edmonton, AB T6G 2E1, Canada 